\title{
Research on the bottom deformation of Vertical Metal Tank Based on finite element analysis
}

\author{
Cun jun $\mathrm{Li}^{1, \mathrm{a}}$, Xian lei Chen ${ }^{1, \mathrm{~b}}$, Hua dong $\mathrm{HaO}^{1, \mathrm{c}}$, Hao lei Shi ${ }^{1, \mathrm{~d}}$ \\ ${ }^{1} 257$ Qiandao Road, xincheng, Zhoushan city, zhejiang province, China \\ Zhoushan Institute of Calibration and Testing for Quality and Technology Supervision \\ alicunjun@sina.com bzschenxl@163.com \\ 'gentlehhd@163.com d181144048@qq.com
}

Keywords: Finite element, simulation analysis, Vertical metal tank, Volume metrology

\begin{abstract}
Vertical metal tank is an important compulsory verification metrology instruments of energy trade transfer in China. The metrology of its capacity is accurate or not, directly affecting economic interests and national metrology reputation at home and abroad. In the paper, through the establishment of 50000m3 vertical metal tank bottom finite element model, the tank weight and the water static pressure conditions are simulated, and vertical metallic bottom stress analysis and research is carried out. Respectively vertical metal tank volume of $1000 \mathrm{~m} 3,5000 \mathrm{~m} 3,10000 \mathrm{~m} 3$ were simulated and analyzed at different heights of the tank water level, summarizing the deformation law of tank bottom. Compared capacity comparison method with geometric measurements method by a volume of 5000m3 vertical metal tanks, the reliability of the simulation conclusion is verified. The empirical formula is to be modified, thereby improving the accuracy of capacity of trade transfer metering and ensuring the safe use of the tanks.
\end{abstract}

\section{Introduction}

Vertical metal tank is an important compulsory verification measuring instruments of energy trade transfer in China. The metrology of its capacity is accurate or not, directly affecting economic interests and national metrology reputation at home and abroad [1,2]. At present, with the continuous progress of science and technology, the accuracy of metrology of vertical metal tank capacity increased gradually, but there are still some factors affecting the accuracy of measurement, which the bottom deformation has considerable influence among the vertical metal tank capacity measuring accuracy.

The bottom deformation of tank has more complex reasons, involving geology , construction, mechanical, thermal, material science and other disciplines, mainly divided into the following categories[3]: (1) The original rules of the tank bottom which has a certain taper into a convex concave surface irregularities after the tank pressure test; (2) After filling oil, the tank foundation is caused by uneven settlement and bottom deformation with the unceasingly change of foundation, causing the bottom change without regularity. So the deformation of tank bottom and the bottom volume capacity table have some change, reducing the accuracy test. Practical method for measuring accurate tank bottom volume to actively explore the domestic and foreign experts and scholars over the years. At present, several liquid storage condition of bottom capacity increment measurement methods have been proposed: geometric method, inverted pot, double digit method. In general, these methods are flawed, to reduce or eliminate for tank bottom deformation caused by the error of tank bottom volume fundamentally. Therefore, this paper established the finite element of vertical metal tank bottom deformation model, carry out the research of vertical metal tank bottom deformation, looking for tank bottom deformation rules and improve the capacity of trade transfer metering accuracy and ensure safe and normal use of tanks. 


\section{Structure analysis of Tank bottom}

Tank size and material parameters. The volume of the vertical metal tank is $50000 \mathrm{~m}^{3}$.Tank size and material parameters are as follows: Tank inner diameter $60 \mathrm{~m}$, height of 20m, 8 laps plates, each circle plate height $2400 \mathrm{~mm}$; concrete ring wall: the thickness of $2000 \mathrm{~mm}$, width of $800 \mathrm{~mm}$; backfill sand: thickness of $2000 \mathrm{~mm}$, diameter $59036 \mathrm{~mm}$, material for gravel. Tank bottom edge of the plate: thickness of $21 \mathrm{~mm}$, width of $2000 \mathrm{~mm}$, tank bottom edge of the plate material is SPV490Q.

Structure and configuration of Tank bottom. Such as the conical vertical metal tank is shown in Fig. 1 is widely used in domestic. It mainly components by tank wall, wind ring, reinforcement, tank bottom plate and concrete ring wall etc. This kind of tank bottom positive conic, surrounded by low, high in the middle, the tank bottom slope is commonly in 1.5\% 3.5\%, it can reduce the pressure on the tank bottom of the liquid increases. As shown in Fig. 2 for the typesetting and construction plans of vertical metal tank bottom , middle part of the bottom plate is called middle plate, peripheral bottom plate called the edge plate or annular plate, according to the GB 50341-2003 decides the arrangement form by the tank type size, manufac-turing process and other factors[4].

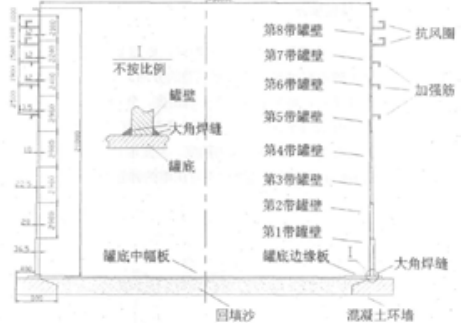

Fig. 1

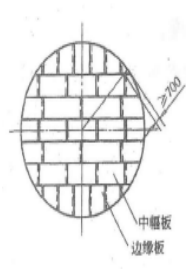

(a)

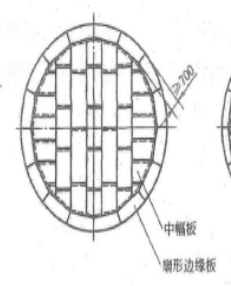

(b)

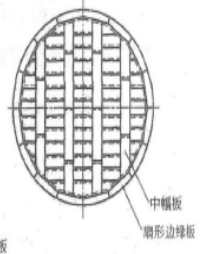

(c)

Fig. 2

Fig. 1 the structure diagram of vertical metal tank which the bottom is conical

Fig. 2 The bottom layout diagram of vertical metal tank

Weld requirements. For welding requirements, each plate (between edge board and the middle board) of the tank bottom can be used lap or welding structure. The repeated bending load is caused by liquid column height, inner pressure and wind load changes, plus the load and foundation subsidence is caused deformation, so the requirement on welding is higher. The bottom of the tank and the tank wall bottom ring of the fillet weld adopt continuous welding, welding height equal to the bottom edge of the plate thickness[5].

\section{Finite Element Modeling and Analysis of Vertical metal tank}

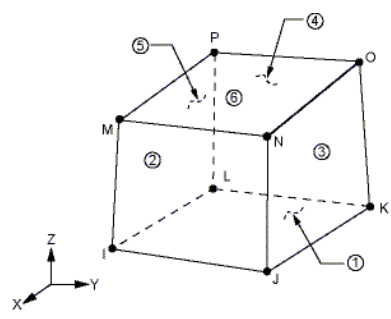

Fig. 3. Solid185 unit

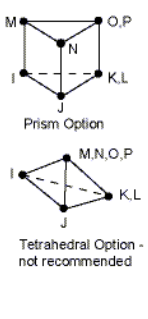

Element selections. When mode the vertical two-dimensional finite element metal tank, it can be

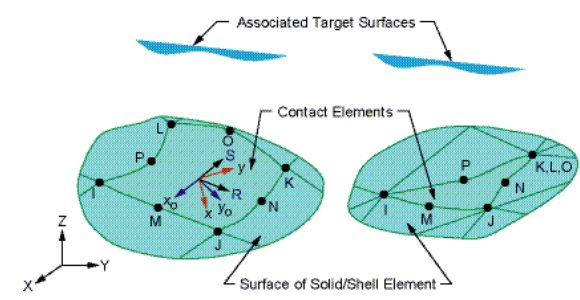

Fig. 4. Conta174 unit used as an axis of symmetry structure, while pressure stress distribution is symmetrical, so the tank and the tank bottom plate can be effectively simulated incompressible elastic-plastic deformation of the material by using a high-order two-dimensional 8-node element Plane183. As shown in Fig. 5 (a), considering the foundation and tank bottom contact and load transmission, establish contact with the foundation model and the tank bottom. Tank bottom is used target element Targe169. The target surface element can apply any translational displacement, angle, temperature, force and moment. On the foundation surface is used contact element Conta172 [6].

Fig. 5 (b) shows, when the three-dimensional modeling, tank and tank bottom plate is used solid element Solid185 (Fig. 3). Between the tank bottom and foundation is used surface-surface contact element. The element Targe170 and the contact element Conta174 are respectively used for the tank 
bottom and on the foundation surface (Fig. 4). As shown in Fig.5 (c), due to 3D modeling unit is too big, reduce element number to reduce hardware requirements, this paper adopts half a tank that is 10 DEG entity modeling.

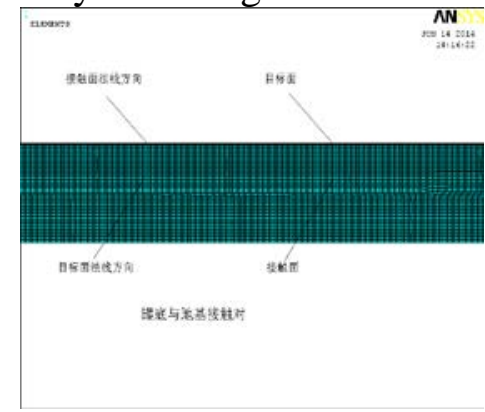

(a)

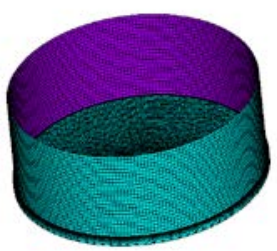

(b)

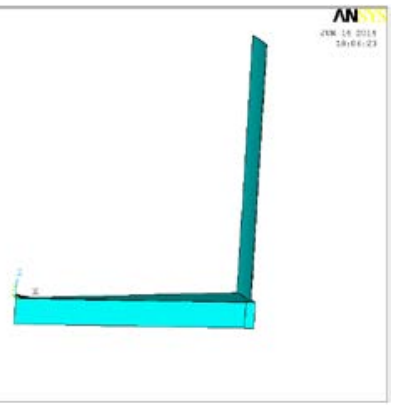

(c)

Fig. 5 tank finite element model

Loads and boundary conditions. When strong water pressure test in this paper, analysis of the stress of tank, as shown in Fig. 6 (a) and (b), respectively, for the 2D model and 3D model of load settings, the tank main load components weight and hydrostatic pressure: (1) By applying a 9.8m/s2 acceleration of gravity to simulate the weight of the tank bottom; (2) Applying a triangular distribution hydrostatic pressure gradually increased from the top to the bottom of the tank wall. According to the formula of hydrostatic pressure, can be calculated the hydrostatic pressure by Pmax $=198000$ Pa from top to bottom triangle distribution of (0-20) $\mathrm{m}$ tank height.

Boundary conditions: (1) Because of unanchored tank model of the horizontal load on friction force constraints, no constraint on the tank, full constraints applied in contact surface with foundation elastic body and the soil; (2) Because of the elastic body and the tank cannot have any displacement in the radial direction, in the elastic body axis section displacement of X axis of zero constraint; (3) Apply ring constraint to the bottom edge plate surface of the outermost node. 4) Symmetry constraint applied to plane of symmetry of 10 DEG modeling.

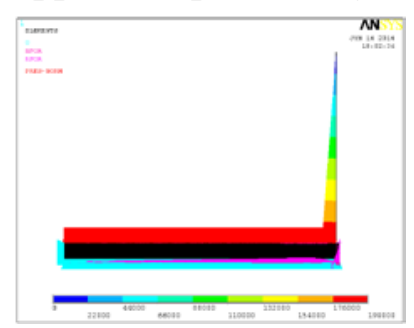

Fig. 6 (a)

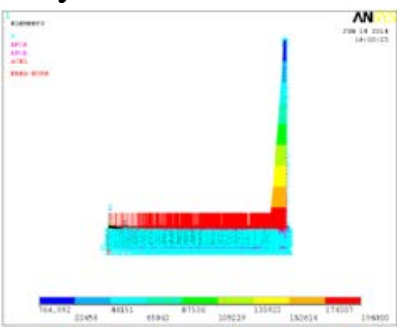

Fig. 6 (b)

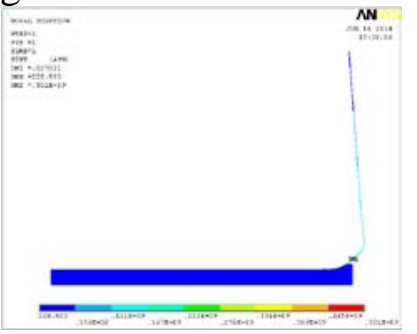

Fig. 7

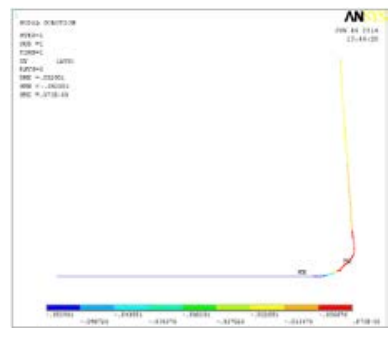

Fig. 8

Fig. 6 schematic diagram of tank in load setting

Fig. 7 tank stress intensity profile

Fig. 8 settlement diagram of tank bottom

Stress analysis of 2D modeling. Simulate 2D tank model, from the tank stress intensity contours in Fig. 7 ,it can be drawn, due to the end effect, the biggest stress intensity appear within the tank bottom and the large fillet weld joint, and gradually reduce from the edge to the center. The maximum stress value is $501 \mathrm{Mpa}$. From settlement of tank bottom figure (Fig. 8) can be concluded that the largest settlement in the middle plate, which is $52.5 \mathrm{~mm}$. It can be seen from the picture in middle plate basic at $52.5 \mathrm{~mm}$, the settlement of settlement is essentially the same in the whole middle plate, namely partial backfill sand in the same settlement. In the concrete ring wall near the gravel on the elastic body, settlement is relatively small for $3.4 \mathrm{~mm}$, but between boundary of backfill sand to the regional subsidence is maximum and deformation gradient speed is fast. It is shown that the amplitude of variation is big and the maximum in this region, and it is more dangerous part of the tank bottom plate.

Stress analysis of 3D modeling. Simulate 3D tank model, from the tank displacement in Fig. 9 can be drawn, the largest settlement in the middle plate, which is $51.6 \mathrm{~mm}$. From large fillet weld press figure (Fig. 10), it can be concluded that large fillet weld pressure is max, it is prone to fatigue 
cracking. From the above Figure show that the 2D and 3D simulation model data is closed, a subtle distinction lies in the degree of density of mesh selection.

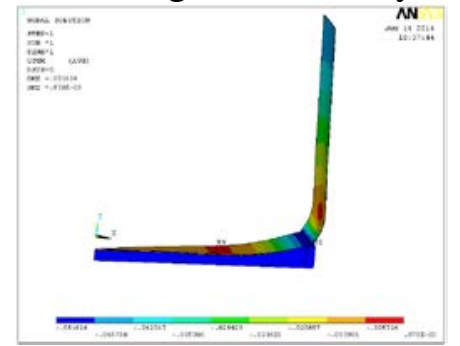

Fig. 9. tank displacement nephogram

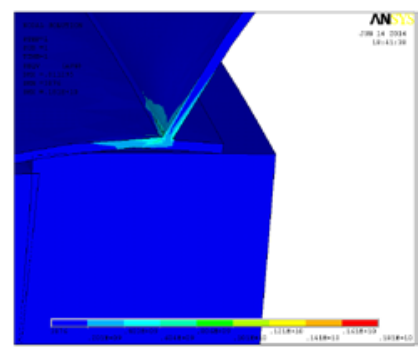

Fig.10. Bigfoot weld pressure nephogram

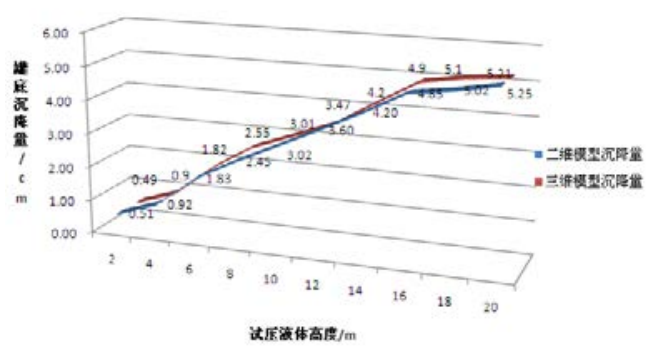

Fig. 11 tank bottom maximum settlement curve in the height of liquid level

As shown in Fig. 11, respectively choosing 2m, 4m, 6m, 8m, 10 m,12 m, 14 m, 16 m, 18 m, 20m height of liquid level of tank bottom to simulate settlement test, the different height of liquid level tank bottom maximum settlement curve is obtained. From the figure, with the liquid height rising, the tank bottom under hydrostatic pressure gradually increases, pressure and settlement of the changes according to certain rules: the settlement rate of tank bottom is most obvious in (4-6) m range, which reached $1 \mathrm{~cm}$; the change is smoothly $\operatorname{In}(6-16) \mathrm{m}$ range and change is about $0.5 \mathrm{~cm}$ in the range of $2 \mathrm{~m}$ internal; the change is rarely in (16-20) $\mathrm{m}$ range, within $0.2 \mathrm{~cm}$.

Based on last section, the vertical metal tank volume of $1000 \mathrm{~m} 3,5000 \mathrm{~m} 3,10000 \mathrm{~m} 3$ were investigated by simulation and data analysis. We can conclude that: 1) when the height of liquid level $\mathrm{H}$ in $(0, \mathrm{H} 1)$ range, the settlement tank plate basically no change; 2$)$ when the height of liquid level $\mathrm{H}$ in the (H1, H2) interval, tank plate to generate uniform linear deformation and settlement; 3) when the height of liquid level $\mathrm{H}$ in the (H2, H3) interval, the settlement tank plate changes little, showing steady state.

\section{Test verification and Experience summary}

Test verification. In this paper, a vertical metal tank which the volume is $5000 \mathrm{~m} 3$ is measured by using capacity comparison method. The standard is ROSE MOUNT Mass Flowmeter that made in American model CMF300M/2400, the accuracy rating of 0.1 through linear fitting, its accuracy can reach 0.5 per thousand. Tank pipeline connection and connection using a pipeline between the test medium tank and the tank by sealing pipeline, with quick joint way, filter installed in the middle. Test medium for tank use hydraulic settlement tests of the pool water.

Test procedure: 1) Check the connection of two tanks pipeline to ensure that the pipeline does not leak, not channeling fluid with other tank, control valve in good condition and flexible operation; 2) Water tank and measured tank connected pipeline to be tested before testing is full of liquid, and emptying pipeline gas; 3) Injection water from water tank to measured tank, estimates height of water injection point position of measured tank by flowmeter cumulant. The injection point are: the first point is the bottom of the tank height, can also be appropriate to go beyond a point, after that every $2 \mathrm{~m}$ a point; 4)Test flow around $70 \mathrm{t} / \mathrm{h}$, two tank water temperature is around 23 degrees temperature, density calculated by pure water density table. Compared capacity comparison method with geometric measurements method by a tank volume every $2 \mathrm{~m}$ a point, get the following table 1 . 
Can be drawn, in (0-0.2) m height, bottom volume which vaule is $0.102 \mathrm{~m} 3$, changed little ; Starting from the $0.5 \mathrm{~m}$ height at the bottom of the mutation $1.239 \mathrm{~m} 3$, produce settlement; in (0.5-8) m height range, bottom height increment with linear state; In (8-15) the height range, bottom increment gradually stabilized. In summary, the measured results and simulation is consistent with the conclusion.

Table 1 tank volume table compared with capacity comparison method and geometric measurements method

\begin{tabular}{ccccc}
\hline Hight $\left(\mathrm{m}^{3}\right)$ & $\begin{array}{c}\text { Volume of geometric measurements } \\
\text { method }\left(\mathrm{m}^{3}\right)\end{array}$ & $\begin{array}{c}\text { Volume of capacity } \\
\text { comparison }\left(\mathrm{m}^{3}\right)\end{array}$ & $\begin{array}{c}\text { D-value } \\
\left(\mathrm{m}^{3}\right)\end{array}$ & $\begin{array}{c}\text { relative error } \\
(\%)\end{array}$ \\
\hline 0.2 & 70.454 & 70.556 & 0.102 & 0.145 \\
0.5 & 163.009 & 163.721 & 0.712 & 0.435 \\
1 & 319.864 & 320.723 & 0.859 & 0.268 \\
2 & 633.341 & 634.519 & 1.178 & 0.186 \\
4 & 1260.585 & 1262.264 & 1.679 & 0.133 \\
6 & 1888.124 & 1890.122 & 1.998 & 0.106 \\
8 & 2515.570 & 2517.769 & 2.199 & 0.087 \\
10 & 3143.099 & 3145.378 & 2.279 & 0.072 \\
12 & 3770.794 & 3773.045 & 2.251 & 0.060 \\
14 & 4398.692 & 4400.981 & 2.289 & 0.052 \\
15 & 4712.672 & 4714.911 & 2.239 & 0.048 \\
\hline
\end{tabular}

Experience summary. The principle of geometric measurement method of Level (or total station) way is as tank bottom a circle, assignment based on tank type to determine the intersection point position and measuring points of concentric circles (I II III,.., m) and radius $(\mathrm{O}-1,2, \ldots, \mathrm{O}-\mathrm{O}-\mathrm{n}$, $\mathrm{n}$ for radius measurement coefficient) on tank bottom. Set up the level in the center of the stable point of tank bottom, one by one with level ruler upright in each measurement point, center of tank bottom point and the measurement datum point, read the readings of the scale, record of each measurement point elevation.

Among them, center distance from concentric circle to the center of tank bottom determine by the equal area circle conditions. The radius of the circle according to the formula (1) calculation. Center distance from concentric circle to the center of tank bottom:

$$
\begin{gathered}
R_{\mathrm{I}}=R \sqrt{1 / m} \\
R=R \sqrt{2 / m} \\
R_{m-1}=R \sqrt{m-1 / m} \\
R_{m}=R
\end{gathered}
$$

In the formula: $\mathrm{R} \longrightarrow$ The first tank plate inner radius, mm;

$M-$ The number of aliquots of the ring.

Measuring tank bottom with geometric measurement method, the highest point of the tank bottom following capacity $\left(\Delta V_{B}\right)$ is calculated as follows:

$$
\begin{aligned}
\Delta V_{B}= & \frac{\pi d^{2}}{4} \times\left\{\frac{1}{3 m n} \sum_{i=1}^{n} F_{0}\left(h_{d}, B_{0, i}, B_{\text {base }}\right)+\frac{7}{6 m n} \sum_{i=1}^{n} F_{1}\left(h_{d}, B_{1, i}, B_{\text {base }}\right)+\right. \\
& \frac{1}{m n} \sum_{i=1}^{n} F_{2}\left(h_{d}, B_{2, i}, B_{\text {base }}\right)+\cdots \cdots+\frac{1}{m n} \sum_{i=1}^{n} F_{m-1}\left(h_{d}, B_{m-1, i}, B_{\text {base }}\right)+ \\
& \left.\frac{1}{2 m n} \sum_{i=1}^{n} F_{m}\left(h_{d}, B_{m, i}, B_{\text {base }}\right)\right\} \times 10^{-6}
\end{aligned}
$$

In the formula: $\Delta V_{B} \longrightarrow$ The bottom of the tank bottom volume, as a function of height $\mathrm{h}_{d}, \mathrm{dm}^{3}$;

$h_{d}$ —Preparation of the height of the bottom volume capacity table (interval as the measurement datum point to highest of tank bottom point), mm; $d$ - The first tank plate inner radius, mm; 


$$
\begin{gathered}
B_{O, i}, B_{l, i}, \ldots, B_{m, i}-\text { Each measuring point elevation, mm; } \\
F\left(h_{d}, B_{m, n}, B_{\text {base }}\right)-\text { The measurement datum elevation, mm; } \\
F\left(h_{d}, B_{m, i}, B_{\text {base }}\right)= \begin{cases}B_{m, i}+h_{d}-B_{\text {base }} & \left(B_{m, i}+h_{d}>B_{\text {base }}\right), \\
0 & \left(B_{m, i}+h_{d} \leq B_{\text {base }}\right)\end{cases}
\end{gathered}
$$

Through the simulation and the measured data, combination of calculation model of JJG168-2005 $<$ volume of vertical metal tank $>$ is to corrected bottom measurement data of the empty tank. Such as volume of $5000 \mathrm{~m} 3$ vertical metal tank in simulation filled with water, the center of the settlement was $12 \mathrm{~m}$, bottom edge settlement was $1.5 \mathrm{~m}$. Through corrected bottom measurement data, get the following table 2:

Table 2 The capacity comparison table of Vertical metal tank geometry measurement method before and after the correction and capacity measurement method

\begin{tabular}{cccccc}
\hline $\begin{array}{c}\text { hight } \\
(\mathrm{m})\end{array}$ & $\begin{array}{c}\text { volume of } \\
\text { geometric } \\
\text { measurements } \\
\text { method }(1)\end{array}$ & $\begin{array}{c}\text { corrected data geometric } \\
\text { measurements method(2) } \\
\left(\mathrm{m}^{3}\right)\end{array}$ & $\begin{array}{c}\text { capacity } \\
\text { comparison } \\
\text { method(3) } \\
\left(\mathrm{m}^{3}\right)\end{array}$ & $\begin{array}{c}\text { Relative error } \\
\text { between } 1 \text { and } \\
3 \\
(\%)\end{array}$ & $\begin{array}{c}\text { Relative error } \\
\text { between 2 and } \\
3 \\
(\%)\end{array}$ \\
\hline 0.2 & 70.454 & 70.62 & 70.556 & 0.145 & 0.091 \\
0.5 & 163.009 & 163.561 & 163.721 & 0.435 & 0.098 \\
1 & 319.864 & 320.41 & 320.723 & 0.268 & 0.098 \\
2 & 633.341 & 634.046 & 634.519 & 0.186 & 0.074 \\
4 & 1260.585 & 1261.609 & 1262.264 & 0.133 & 0.052 \\
6 & 1888.124 & 1889.466 & 1890.122 & 0.106 & 0.035 \\
8 & 2515.570 & 2517.231 & 2517.769 & 0.087 & 0.021 \\
10 & 3143.099 & 3145.078 & 3145.378 & 0.072 & 0.010 \\
12 & 3770.794 & 3773.092 & 3773.045 & 0.060 & 0.001 \\
14 & 4398.692 & 4401.308 & 4400.981 & 0.052 & 0.007 \\
15 & 4712.672 & 4715.448 & 4714.911 & 0.048 & 0.011 \\
\hline
\end{tabular}

It can be obtained from Table 2, the corrected volume of tank data compare with capacity comparison method, it relative error compare with the uncorrected were less than $50 \%$, and the relative error is less than $0.1 \%$.

\section{Conclusion and Prospect}

In this paper, through the research on static strength of two and 3D finite element model of vertical metal tank which the volume is $50000 \mathrm{~m} 3$, the following conclusions are obtained:(1) By using the elastic body modeling, the deformation in the ground is true reflected; (2)By two and three-dimensional finite element model of simulation, the maximum stress intensity values of the tank occurred in the fillet weld inner weld seam and tank bottom junction. The largest settlement is located in the web plate, and the bottom plate settlement remained unchanged; (3) Through simulating tank bottom settlement in different liquid height, the two and 3D tank bottom settlement curve is get, analysing the settlement changes at each height. The simulation data are analyzed, the reliability of simulation conclusion is verified by comparing the capacity comparison method and the geometric measurement method, the result is revised through empirical formula. But the research is still not perfect, the vertical metal tanks which have different volumes and different foundations are further simulated, getting a set of detailed simulation data, and to find and summarize the tank bottom deformation rules, and then enhanced the capacity of trade transfer accuracy and ensured the normal use of tank safety by help. 


\section{Acknowledgment}

The research is supported by the Special Fund Project of AQSIQ for the Scientific Research of Public Welfare Industry (201210106) and science and technology and public technology research and social development project of Zhejiang Provincial Department (2012C33082). The authors would like to express a special thank for its support.

\section{References}

[1] Z.Y. Liu. Volume metrology.China Metrology Publishing House,2009. In Chinese.

[2] X.L. Chen, H.D. Hao, H.J. Li, B. Chen, H.N. Men. Study on 3D Laser Scanner Method on Volume Metrology of Large-scale Vertical Tank. China Measurement \& Test,2014, 40(2): 44-48.In Chinese.

[3] Y.F. Yang, W.S. Wang. Effect of bottom deformation of vertical drum on its volumety. Refining and Chemical Industry, 2007, 18 (2): 26-28. In Chinese.

[4] GB 50341-2003<<Code for design of vertical cylindrical welded steel oil tanks >>. Beijing: China National Petroleum Corporation, 2003. In Chinese.

[5] L. Li. Numerical analysis of large (super) vertical crude oil storage tank foundations. Harbin Institute of Technology, 2008. In Chinese.

[6] J.C. Zhao. Finite element analysis of large vertical oil tank based on contact simulation of the interaction between tank bottom plate and foundation. Beijing University of Chemical Technology, 2008. In Chinese. 